\title{
71. On Conformal Mapping of Polygonal Domains
}

\author{
By Yûsaku Komatu \\ Department of Mathematics, Tokyo Institute of Technology, Tokyo \\ (Comm. by Z. Suetuna, M.J.A., June 12, 1957)
}

1. In a recent paper an explicit formula has been established for an analytic function mapping an annulus onto a domain which contains the point at infinity in its interior and whose boundary consists of two rectilinear polygons (see Satz 8 in [2]). Since the formula has been obtained there as a corollary of a general representation theorem for analytic functions in which the Villat-Stieltjes formula has been taken into account as an essential tool for its proof, it has been preannounced that another more direct way of proof will be published later. In fact, it is possible to give an alternative proof without any reference to the Villat-Stieltjes representation formula.

Main purpose of the present paper is to fulfil the promise mentioned above. The present method is of primitive nature and is really an analogue of a classical proof in establishing the Schwarz-Christoffel formula for a function mapping a circle onto the exterior of a polygon. Moreover, a related formula for a function mapping an annulus onto a rectilinear polygonal ring domain which does not contain the point at infinity has been already derived by a method which remains valid in the present case with less modification (cf. the proof of Theorem 4 in [1]).

On the other hand, the paper [1] contains further results on mappings onto multiply connected circular polygonal domains. Supplementary remarks will be made below also to some of them.

2. Now an alternative proof will be given below for the theorem in question (Satz 8 in [2]) which may be re-stated as follows:

THEOREM 1. Let $f(z)$ be an analytic function which maps an annulus $(0<) q<|z|<1$ onto a rectilinear polygonal ring domain containing the point at infinity in its interior. Let the vertices of the boundary polygons be designated by $f\left(e^{i \varphi_{\mu}}\right)(\mu=1, \cdots, m)$ and $f\left(q e^{i \psi_{\nu}}\right)$ $(\nu=1, \cdots, n)$ and the interior angles (with respect to the image-domain) at these vertices by $\gamma_{\mu} \pi$ and $\delta_{\nu} \pi$, respectively. Further, let $z_{\infty}$ be the antecedent of the point at infinity. Then there holds a formula

$$
f(z)=C \int^{z} z^{i c^{*-1}} \frac{\prod_{\mu=1}^{m} \sigma\left(i \lg z+\varphi_{\mu}\right)^{\gamma_{\mu}-1} \prod_{\nu=1}^{n} \sigma_{3}\left(i \lg z+\psi_{\nu}\right)^{\delta_{\nu-1}}}{\sigma\left(i \lg \left(z / z_{\infty}\right)\right)^{2} \sigma\left(i \lg \left(\bar{z}_{\infty} z\right)\right)^{2}} d z+C^{\prime}
$$

with certain constants $C$ and $C^{\prime}$. Here the sigma-functions are those 
of Weierstrass concerning the primitive quasi-periods $2 \omega_{1}=2 \pi$ and $2 \omega_{3}=-2 i \lg q$ and $c^{*}$ is a real constant defined by

$$
c^{*}=\frac{\eta_{1}}{\pi}\left(\sum_{\mu=1}^{m}\left(1-\gamma_{\mu}\right) \varphi_{\mu}+\sum_{\nu=1}^{n}\left(1-\delta_{\nu}\right) \psi_{\nu}+4 \arg z_{\infty}\right) .
$$

Further there exist monodromy conditions

$$
\begin{gathered}
\sum_{\mu=1}^{m}\left(\gamma_{\mu}-1\right)\left(\zeta\left(i \lg z_{\infty}+\varphi_{\mu}\right)-\frac{\eta_{1}}{\pi} \varphi_{\mu}\right)+\sum_{\nu=1}^{n}\left(\delta_{\nu}-1\right)\left(\zeta_{3}\left(i \lg z_{\infty}+\psi_{\nu}\right)-\frac{\eta_{1}}{\pi} \psi_{\nu}\right) \\
-2\left(\zeta\left(2 i \lg \left|z_{\infty}\right|\right)-\frac{2 \eta_{1}}{\pi} \arg z_{\infty}\right)=0
\end{gathered}
$$

and

$$
\int_{0}^{2 \pi} e^{-c_{\varphi} \psi_{\varphi}} \frac{\prod_{\mu=1}^{m} \sigma\left(\varphi_{\mu}-\varphi\right)^{\gamma_{\mu}-1} \prod_{\nu=1}^{n} \sigma_{3}\left(\psi_{\nu}-\varphi\right)^{\delta_{\nu}-1}}{\left|\sigma\left(\varphi+i \lg z_{\infty}\right)\right|^{4}} d \varphi=0
$$

which must be satisfied by several preassigned quantities.

Proof. It is evident that the mapping function $f(z)$ can be prolonged by means of successive inversions to a function which is analytic over the pricked plane $0<|z|<\infty$ save possibly at the points $e^{i \varphi_{\mu}}(\mu=1, \cdots, m)$ and $q e^{i \psi_{\nu}}(\nu=1, \cdots, n)$. The prolonged function which will be again denoted by $f(z)$ is, in general, infinitely many-valued and possesses logarithmic singularities at $e^{i \varphi_{\mu}}$ and $q e^{i \psi_{\nu}}$ as well as their equivalents provided $\gamma_{\mu}$ and $\delta_{\nu}$, respectively, are irrational, while otherwise the singularities are algebraic. Now, in view of the piecewise rectilinearity of the boundary of the image-domain, any successive inversions repeated even times cause a motion of the image-domain. Hence, introducing a variable $Z$ and its function $F(Z)$ by

$$
Z=i \lg z, \quad F(Z)=f\left(e^{-i Z}\right)(=f(z)),
$$

$F(Z)$ possesses a linear polymorphic character represented by

$$
F(Z+2 \pi)=g_{0} F(Z)+h_{0}, \quad F(Z-2 i \lg q)=g F(Z)+h
$$

where $g_{0}, h_{0}, g$ and $h$ are certain constants with

$$
\left|g_{0}\right|=1 \text { and }|g|=1 \text {, }
$$

depending on the order of the corresponding prolongations. The differential expression $F^{\prime \prime}(Z) / F^{\prime}(Z)$ is, consequently, one-valued throughout the whole finite plane $|Z|<\infty$ and possesses, moreover, an automorphic character represented by

$$
\frac{F^{\prime \prime}(Z+2 \pi)}{F^{\prime}(Z+2 \pi)}=\frac{F^{\prime \prime}(Z)}{F^{\prime}(Z)}, \quad \frac{F^{\prime \prime}(Z-2 i \lg q)}{F^{\prime}(Z-2 i \lg q)}=\frac{F^{\prime \prime}(Z)}{F^{\prime}(Z)} .
$$

It is further shown that $F^{\prime \prime}(Z) / F^{\prime}(Z)$ is really meromorphic throughout $|Z|<\infty$. In fact, the local developments of $f(z)$ about critical points $e^{i \varphi_{\mu}}$ and $q e^{i \psi_{\nu}}$ are of the form

and

$$
f(z)=f\left(e^{i \varphi_{\mu}}\right)+s_{\mu}\left(z-e^{i \rho_{\mu}}\right)^{r_{\mu}}+\cdots \quad\left(s_{\mu} \neq 0\right)
$$

$$
f(z)=f\left(q e^{i \psi_{\nu}}\right)+t_{\nu}\left(z-q e^{i \psi_{\nu}}\right)^{\delta \nu}+\cdots \quad\left(t_{\nu} \neq 0\right)
$$

respectively. Hence $F(Z)$ is developed about $-\varphi_{\mu}$ and $-\psi_{\nu}+i \lg q$ in the form 
and

$$
F(Z)=F\left(-\varphi_{\mu}\right)+s_{\mu}\left(-i e^{i \varphi_{\mu}}\right)^{\gamma_{\mu}}\left(Z+\varphi_{\mu}\right)^{\gamma_{\mu}}+\cdots
$$

$$
F(Z)=F\left(-\psi_{\nu}+i \lg q\right)+t_{\nu}\left(-i q e^{i \psi_{\nu}}\right)^{\delta_{\nu}}\left(Z+\psi_{\nu}-i \lg q\right)^{\delta_{\nu}}+\cdots,
$$

whence readily follow

$$
\frac{F^{\prime \prime}(Z)}{F^{\prime}(Z)}=\frac{\gamma_{\mu}-1}{Z+\varphi_{\mu}}+\cdots, \quad \frac{F^{\prime \prime}(Z)}{F^{\prime}(Z)}=\frac{\delta_{\nu}-1}{Z+\psi_{\nu}-i \lg q}+\cdots,
$$

respectively, showing that $F^{\prime \prime}(Z) / F^{\prime}(Z)$ possesses simple poles with residues $\gamma_{\mu}-1$ and $\delta_{\nu}-1$ at $-\varphi_{\mu}$ and $-\psi_{\nu}+i \lg q$, respectively. Finally, since $f(z)$ possesses simple poles at $z_{\infty}$ as well as at $q^{2} / \bar{z}_{\infty}$ it will be similarly verified, starting from the local developments

$$
f(z)=\frac{s}{z-z_{\infty}}+\cdots \quad(s \neq 0) \text { and } f(z)=\frac{t}{z-q^{2} / \bar{z}_{\infty}}+\cdots \quad(t \neq 0)
$$

that $F^{\prime \prime}(Z) / F^{\prime}(Z)$ possesses simple poles with residue -2 at

$$
Z_{\infty}=i \lg z_{\infty} \quad \text { and } \quad 2 i \lg q+\bar{Z}_{\infty}=i \lg \left(q^{2} / \bar{z}_{\infty}\right) \text {. }
$$

Thus it has been shown that $F^{\prime \prime} / F^{\prime}(Z)$ is really an elliptic function of order $m+n+2$ with primitive periods $2 \pi$ and $-2 i \lg q$. Its irreducible poles are all simple and lie at $-\varphi_{\mu},-\psi_{\nu}+i \lg q, Z_{\infty}$ and $2 i \lg q$ $+\bar{Z}_{\infty}$, the residues being $\gamma_{\mu}-1, \delta_{\nu}-1,-2$ and -2 , respectively. It is noted by the way that the sum of these residues, of course, surely vanishes; in fact, there holds moreover

$$
\sum_{\mu=1}^{m}\left(\gamma_{\mu}-1\right)=\sum_{\nu=1}^{n}\left(\delta_{\nu}-1\right)=2 .
$$

Now, based on a well-known theorem in the theory of elliptic functions, $F^{\prime \prime}(Z) / F^{\prime}(Z)$ can be represented in terms of zeta-functions in the form

$$
\begin{aligned}
\frac{F^{\prime \prime}(Z)}{F^{\prime}(Z)}=\sum_{\mu=1}^{m}\left(\gamma_{\mu}-1\right) \zeta\left(Z+\varphi_{\mu}\right) & +\sum_{\nu=1}^{n}\left(\delta_{\nu}-1\right) \zeta\left(Z+\psi_{\nu}-i \lg q\right) \\
& -2 \zeta\left(Z-Z_{\infty}\right)-2 \zeta\left(Z-\bar{Z}_{\infty}-2 i \lg q\right)+c \\
=\sum_{\mu=1}^{m}\left(\gamma_{\mu}-1\right) \zeta\left(Z+\varphi_{\mu}\right) & +\sum_{\nu=1}^{n}\left(\delta_{\nu}-1\right) \zeta_{3}\left(Z+\psi_{\nu}\right) \\
& -2 \zeta\left(Z-Z_{\infty}\right)-2 \zeta\left(Z-\bar{Z}_{\infty}\right)+c^{*}
\end{aligned}
$$

$c$ and $c^{*}$ being constants. In view of

$$
\frac{F^{\prime \prime}(Z)}{F^{\prime}(Z)}=-i\left(1+z \frac{f^{\prime \prime}(z)}{f^{\prime}(z)}\right) \quad(Z=i \lg z, F(Z)=f(z)),
$$

the last equation becomes, after returning to the original variables,

$$
\begin{gathered}
1+z \frac{f^{\prime \prime}(z)}{f^{\prime}(z)}=i \sum_{\mu=1}^{m}\left(\gamma_{\mu}-1\right) \zeta\left(i \lg z+\varphi_{\mu}\right)+i \sum_{\nu=1}^{n}\left(\delta_{\nu}-1\right) \zeta_{3}\left(i \lg z+\psi_{\nu}\right) \\
-2 i\left(\zeta\left(i \lg \left(z / z_{\infty}\right)\right)+\zeta\left(i \lg \left(\bar{z}_{\infty} z\right)\right)\right)+i c^{*}
\end{gathered}
$$

whence readily follows the desired representation for $f(z)$. It remains therefore only to determine the value of $c^{*}$. For that purpose it may be remembered that there hold the relations 


$$
\left|\frac{F^{\prime}(Z+2 \pi)}{F^{\prime}(\bar{Z})}\right|=1, \quad\left|\frac{F^{\prime}(Z-2 i \lg q)}{F^{\prime}(Z)}\right|=1 .
$$

But the above expression for $F^{\prime \prime}(Z) / F^{\prime}(Z)$ implies, after integration,

$$
F^{\prime}(Z)=-i C e^{e^{*} z} \frac{\prod_{\mu=1}^{m} \sigma\left(Z+\varphi_{\mu}\right)^{\gamma_{\mu}-1} \prod_{\nu=1}^{n} \sigma_{3}\left(Z+\psi_{\nu}\right)^{\delta_{\nu}-1}}{\sigma\left(Z-Z_{\infty}\right)^{2} \sigma\left(Z-\bar{Z}_{\infty}\right)^{2}},
$$

$C$ being an integration constant, whence follow the relations

$$
\begin{aligned}
& F^{\prime}(Z+2 \pi)=(-1)^{m} \exp \left(2 \pi c^{*}+2\right. \eta_{1}\left(\sum_{\mu=1}^{m}\left(\gamma_{\mu}-1\right) \varphi_{\mu}\right. \\
& F^{\prime}(Z)\left.\left.+\sum_{\nu=1}^{n}\left(\delta_{\nu}-1\right) \psi_{\nu}+2\left(Z_{\infty}+\bar{Z}_{\infty}\right)\right)\right), \\
& \frac{F^{\prime}(Z-2 i \lg q)=\exp \left(-2 i \lg q \cdot c^{*}\right.}{}+2 \eta_{3}\left(\sum_{\mu=1}^{m}\left(\gamma_{\mu}-1\right) \varphi_{\mu}\right. \\
&\left.\left.F^{\prime}(Z) \quad+\sum_{\nu=1}^{n}\left(\delta_{\nu}-1\right) \psi_{\nu}+2\left(Z_{\infty}+\bar{Z}_{\infty}\right)\right)\right) ;
\end{aligned}
$$

here the equation $\sum_{\mu=1}^{m}\left(\gamma_{\mu}-1\right)+\sum_{\nu=1}^{n}\left(\delta_{\nu}-1\right)=4$ is taken into account. Since $\eta_{3}$ is purely imaginary, the second relation shows that $c^{*}$ must be real. Since $\eta_{1}$ is real, the first relation then gives

$$
c^{*}=\frac{\eta_{1}}{\pi}\left(\sum_{\mu=1}^{m}\left(1-\gamma_{\mu}\right) \varphi_{\mu}+\sum_{\nu=1}^{n}\left(1-\delta_{\nu}\right) \psi_{\nu}-4 \Re Z_{\infty}\right)
$$

with

$$
\Re Z_{\infty}=\Re\left(i \lg z_{\infty}\right)=-\arg z_{\infty} .
$$

Finally, the representation for $f(z)$ having been once established, the monodromy conditions can be verified quite as in [2]. Thus the proof of the theorem has been completed.

It would be noticed that the points $z_{\infty}$ and $q^{2} / \bar{z}_{\infty}$, the poles of $f(z)$, affect to the integral representation for $f(z)$ as if they were vertices with a negative interior angle, i.e. $-\pi$.

3. In any univalent conformal mapping for which the imagedomain contains the point at infinity, there appears correspondingly a simple pole of the mapping function. The existence of a simple pole, however, gives arise no corresponding singularity for the Schwarzian derivative of the function. In fact, this functional remains invariant under any linear transformation applied to the argument function. Consequently, any theorem concerning univalent conformal mapping will remain valid even when the image contains the point at infinity provided the mapping function appears exclusively as Schwarzian derivative.

For instance, theorems concerning mappings from (multiply connected) circular domains onto circular polygonal domains obtained in a previous paper (Theorem 1 and its specialized Theorem 3 in [1]) are 
surely of this category. Here, as an example, the latter will be reformulated:

THEOREM 2. Any function mapping an annulus $q<|z|<1$ conformally and univalently onto a ring domain which is bounded by two circular polygons and may contain the point at infinity in its interior satisfies a differential equation of the third order

$$
\{f(z), z\} \equiv \frac{f^{\prime \prime \prime}(z)}{f^{\prime}(z)}-\frac{3}{2}\left(\frac{f^{\prime \prime}(z)}{f^{\prime}(z)}\right)^{2}=\frac{E(i \lg z)}{z^{2}},
$$

$E(Z)$ being an elliptic function with primitive periods $2 \pi$ and $-2 i \lg q$ (or being a constant). Let $e^{i \varphi_{\mu}}(\mu=1, \cdots, m)$ and $q e^{i \psi_{\nu}}(\nu=1, \cdots, n)$ be the points corresponding to vertices of the boundary polygons where the interior angles (with respect to the image-domain) are $\gamma_{\mu} \pi$ and $\delta_{\nu} \pi$, respectively. Then the function $E(Z)$ possesses its irreducible poles at $-\varphi_{\mu}$ and at $-\psi_{\nu}+i \lg q$, both being of order at most two, and further it satisfies the limit relations

$$
\lim _{Z \rightarrow-\varphi_{\mu}}\left(Z+\varphi_{\mu}\right)^{2} E(Z)=\frac{1-\gamma_{\mu}^{2}}{2}, \quad \lim _{Z \rightarrow-\psi_{\nu}+i \lg _{g}}\left(Z+\psi_{\nu}-i \lg q\right)^{2} E(Z)=\frac{1-\delta_{\nu}^{2}}{2} .
$$

Here it would be remembered again that a simple pole of $f(z)$ behaves as if it were a vertex with interior angle $-\pi$ and correspondingly that $\left(1-\alpha^{2}\right) / 2$ vanishes really for $\alpha=-1$.

\section{References}

[1] Komatu, Y., Conformal mapping of polygonal domains. Journ. Math. Soc. Japan 2 (1950), 133-147; a preliminary report under the same title was published in Kōdai Math. Sem. Rep. Nos. $3 / 4$ (1949), 47-50.

[2] Komatu, Y., Integraldarstellungen für gewisse analytische Funktionen nebst den Anwendungen auf konforme Abbildung. Kōdai Math. Sem. Rep. 9 (1.957). (in press) 